\title{
Model of charge-state distributions for electron cyclotron resonance ion source plasmas
}

\author{
D. H. Edgell,* J. S. Kim, and S. K. Wong \\ FARTECH, Inc., 3146 Bunche Avenue, San Diego, California 92122 \\ R. C. Pardo and R. Vondrasek \\ Physics Division, Argonne National Laboratory, Argonne, Illinois 60439
}

(Received 4 June 1999; published 20 December 1999)

\begin{abstract}
A computer model for the ion charge-state distribution (CSD) in an electron cyclotron resonance ion source (ECRIS) plasma is presented that incorporates non-Maxwellian distribution functions, multiple atomic species, and ion confinement due to the ambipolar potential well that arises from confinement of the electron cyclotron resonance (ECR) heated electrons. Atomic processes incorporated into the model include multiple ionization and multiple charge exchange with rate coefficients calculated for non-Maxwellian electron distributions. The electron distribution function is calculated using a FokkerPlanck code with an ECR heating term. This eliminates the electron temperature as an arbitrary user input. The model produces results that are a good match to CSD data from the ANL-ECRII ECRIS. Extending the model to 1D axial will also allow the model to determine the plasma and electrostatic potential profiles, further eliminating arbitrary user input to the model.
\end{abstract}

PACS numbers: 29.25.Ni, 52.65.Kj, 52.65.Ff

\section{INTRODUCTION}

The complete understanding and optimization of an electron cyclotron resonance ion source (ECRIS) is complicated with many issues to consider, such as plasma confinement, neutrals, multiple atomic species, and the electron cyclotron resonance heating (ECRH). Optimization for higher charge states and higher current with low emittance is challenging. A typical optimization is by trial and error because there are few suitable numerical tools available, none with a comprehensive modeling capability or that rely solely on experimental "knobs" as input.

Typical ECRIS charge-state distribution (CSD) modeling has been a $0 \mathrm{D}$ fluid model [1-3], where plasma parameters are assumed to be uniform over the plasma volume and, thus, no spatial effects are considered. The ion CSD is determined by solving a set of coupled fluid equations. Confinement is determined from a simple potential/magnetic well model. This OD fluid modeling has several drawbacks, in particular, the neglect of the nonMaxwellian nature of the electron distribution function, plus a reliance on arbitrary user inputs.

Because of ECRH and mirror confinement, the electrons in an ECRIS are expected to be highly nonMaxwellian and nonisotropic. The electrons in most ECRIS models are typically treated as two separate species, cold or warm Maxwellian electrons and hot well electrons that can be modeled either as Maxwellian or as collisionless and perfectly confined. The temperatures of both electron species need to be input. The electron confinement usually ignores the potential between the plasma

*Email address: edgell@far-tech.com and the wall despite evidence that is comparable to the cold electron energy [4].

The actual electron distribution function $f_{e}$ is better modeled by a single continuous non-Maxwellian, nonisotropic distribution function. A Fokker-Planck code, such as that presented in this paper, determines $f_{e}$ taking into account rf heating, and confinement by both the magnetic field and the electrostatic potential.

Typical ECRIS models are not strictly predictive in nature. That is, they rely on inputs that are arbitrarily adjusted by the user until a match is found for an experimentally measured CSD. Adjustable inputs can include the electron density, electron temperature, hot to cold electron ratio, the depth of the electrostatic well, etc.

Ideally, an ECRIS model should require as input parameters only ion source design parameters and experimental knobs such as the magnetic field, gas inlet flux, rf power, etc. Such a code, relying on no arbitrary user input, could be considered truly predictive in nature. The Fokker-Planck electron modeling presented here eliminates the electron temperature as an arbitrary input in favor of the electron cyclotron rf (ECRF) input power.

In this paper, we present initial results of our ECRIS modeling code which attempts to improve ECRIS modeling by using an electron Fokker-Planck code.

\section{ECRIS MODEL}

First, we discuss the atomic collisions and cross sections incorporated into the model. Next, we describe the conventional OD fluid model that was used as a starting point for our model. In the last sections, we introduce the addition of a Fokker-Planck electron code to improve the model. 


\section{A. Collisional processes}

Collisional processes incorporated into the model so far include the single, double, and triple electron impact ionization along with single, double, triple, and quadruple charge exchange (CX).

For single ionization, the empirical cross sections of either Lotz [5] or Müller et al. [6] can be used. Multipleionization cross sections $\sigma_{j, q}^{k I}$ are taken to follow the empirical formula of Müller et al. [6,7].

Single, double, triple, and quadruple charge exchange use the empirical cross section scaling laws of Müller and Salzborn [8].

For simplicity, we will include only single-step ionization/charge-exchange collision terms in all of the equations to follow.

\section{B. OD fluid model}

First, we describe a conventional 0D fluid model that was used as a starting point for our ECRIS modeling. Throughout this paper, the subscript $j$ will refer to the ion atomic species and the subscript $q$ to the ion charge.

\section{Neutral modeling}

The multispecies OD neutral density inside the plasma $n_{j, 0}$ is determined from the neutral density outside the plasma $n_{j, g}$ by balancing the net neutral flux across the plasma surface and the rate at which neutrals are converted into ions inside the plasma volume [1]:

$$
\begin{aligned}
0= & 0.5 A_{p} v_{j, 0}\left(n_{j, g}-n_{j, 0}\right) \\
& -V_{p} n_{j, 0}\left(n_{e}\langle\sigma v\rangle_{j, 0}^{I}+\sum_{j_{2}} \sum_{q_{2}=1}^{A_{j_{2}}}\left[n_{j_{2}, q_{2}}\langle\sigma v\rangle_{j_{2}, q_{2} \rightarrow j}^{\mathrm{CX}}\right]\right) \\
& +V_{p} n_{j, 1} \sum_{j_{2}} n_{j_{2}, 0}\langle\sigma v\rangle_{j, 1 \rightarrow j_{2}}^{\mathrm{CX}},
\end{aligned}
$$

where $n_{j, q}$ is the density of ions of atomic species $j$ and charge $q, A_{p}$ is the plasma surface area, and $V_{p}$ is the plasma volume. This balance equation can be readily solved for $n_{j, 0}$.

The neutral temperature $T_{j, 0}$ is assumed to be a constant determined by the neutral source. In actuality, there is a population of neutrals at the ion temperature $T_{i}$ due to charge exchange that we ignore.

\section{Electron modeling and quasineutrality}

In the OD fluid model, the electrons are treated as two separate cold and hot electron populations. The cold electron temperature $T_{e c}$ and hot electron temperature $T_{e h}$ are considered constant input parameters.

The ratio of the two populations

$$
R_{e}=\frac{n_{e h}}{n_{e c}}
$$

is also a constant input parameter. The electron densities are determined by the quasineutrality condition

$$
n_{e}=n_{e c}\left(1+R_{e}\right)=\sum_{j} \sum_{q=1}^{A_{j}} q n_{j, q} .
$$

The global electron particle confinement time $\tau_{e}^{P}$ is

$$
\tau_{e}^{P}=n_{e}\left(\frac{n_{e c}}{\tau_{e c}^{P}}+\frac{n_{e h}}{\tau_{e h}^{P}}\right)^{-1}=\left(1+R_{e}\right)\left(\frac{1}{\tau_{e c}^{P}}+\frac{R_{e}}{\tau_{e h}^{P}}\right)^{-1},
$$

where [9]

$$
\tau_{e c, h}^{P}=1.48 \frac{\ln R_{m}+\sqrt{\ln R_{m}}}{\nu_{e c, h}}
$$

is the particle confinement time of the cold or hot electrons in a mirror field, ignoring any potential confinement, $\nu_{e c, h}$ is the total collision frequency of the cold or hot electrons, and $R_{m}$ is the mirror ratio. Collisions with electrons, ions, and neutrals are included.

\section{Ion confinement and ambipolarity}

The particle confinement time $\tau_{j, q}^{P}$ for an ion of atomic species $j$ and charge $q$, in a confining potential, is given by [10]

$$
\begin{aligned}
\tau_{j, q}^{P}=[ & R_{m} L_{p}\left(\frac{\pi m_{j}}{2 k_{B} T_{i}}\right)^{1 / 2} \\
& \left.+\frac{G x^{2}}{\left(x+\frac{1}{2}\right)\left(\nu_{j, q}^{o}+\nu_{j, q}^{i}\right)}\right] \exp (x),
\end{aligned}
$$

where $\nu^{o}$ and $\nu^{i}$ denote the ion collision frequency with neutrals and other ions, respectively, and

$$
x=\frac{q e \varphi_{w}}{T_{i}} \quad \text { and } \quad G=\frac{\sqrt{\pi}\left(R_{m}+1\right) \ln \left(2 R_{m}+2\right)}{2 R_{m}},
$$

where $\varphi_{w}$ is the ion confining well potential and $L_{p}$ is the length of the core plasma. Radial transport is assumed to be negligible compared to axial end loss.

In the steady state, the electron and ion end loss currents must balance for ambipolarity.

$$
\frac{n_{e}}{\tau_{e}^{P}}-S_{e}^{\mathrm{ext}}=\sum_{j} \sum_{q=1}^{A_{j}} \frac{q n_{j, q}}{\tau_{j, q}^{P}},
$$

where $S_{e}^{\text {ext }}$ is the external electron source. An external ion source, such as from the first stage of a two-stage ECRIS, could easily be added to Eq. (5). This ambipolarity condition along with Eq. (4) can be solved for the central well potential $\varphi_{w}$.

\section{Ion power balance}

The ion temperature is determined by solving the ion power balance

$$
\frac{d}{d t}\left(\frac{3}{2} T_{i} \sum_{j} n_{i}\right)=\sum_{j}\left(p_{j}^{o}+p_{j}^{e i}+p_{j}^{\mathrm{CX}}+p_{j}^{\text {loss }}\right),
$$


where the terms on the right-hand side account for the ion energy sources and sinks that are described below. All ion species are assumed to have the same temperature. Model test runs with separate ion temperatures, for plasmas similar to those discussed in Sec. III B, have resulted in temperatures that differ by less than $1 \%$.

Initial ion energy. The ions start with the initial temperature of the neutral atoms $T_{j, 0}$,

$$
p_{j}^{o}=\frac{3}{2} T_{j, 0} n_{j, 0}\left\{n_{e}\langle\sigma v\rangle_{j, 1}^{I}+\sum_{j_{2}} \sum_{q_{2}=1}^{A_{j_{2}}}\left[n_{j_{2}, q_{2}}\langle\sigma v\rangle_{j_{2}, q_{2} \rightarrow j}^{\mathrm{CX}}\right]\right\} .
$$

Electron collisional energy exchange. The ions are heated by collisions with the electrons,

$$
p_{j}^{e i}=\frac{3}{2} n_{e}\left(T_{e}-T_{i}\right) \sum_{q=1}^{A_{j}} \nu_{j, q}^{\mathrm{Ee}},
$$

where $\nu_{j, q}^{\mathrm{Ee}}$ is the energy exchange frequency for collisions between an ion and the electron background.

Charge-exchange energy loss. Ions that are neutralized by charge exchange leave the plasma taking their energy with them,

$$
p_{j}^{\mathrm{CX}}=-\frac{3}{2} T_{i} n_{j, 1} \sum_{j_{2}}\left[\langle\sigma v\rangle_{j, k \rightarrow j_{2}}^{\mathrm{CX}} n_{j_{2}, 0}\right] .
$$

Endloss energy. The ions that escape the potential well carry an average energy $\bar{E}_{j, q}^{\text {loss }}$ away from the plasma,

$$
p_{j}^{\text {loss }}=-\sum_{q=1}^{A_{j}} \frac{n_{j, q} \bar{E}_{j, q}^{\text {loss }}}{\tau_{j, q}^{P}} .
$$

\section{Ion CSD modeling}

The ion CSD is arguably the most important result desired from an ECRIS model. Traditionally, ECRIS models have determined the CSD by solving a coupled set of fluid equations for multiple atomic species,

$$
\begin{aligned}
\frac{d n_{j, 1}}{d t}= & \left(n_{j, 0}\langle\sigma v\rangle_{j, 0}^{I}-n_{j, 1}\langle\sigma v\rangle_{j, 1}^{I}\right) n_{e} \\
& +n_{j, 2} \sum_{j_{2}}\left[\langle\sigma v\rangle_{j, 2 \rightarrow j_{2}}^{\mathrm{CX}} n_{j_{2}, 0}\right] \\
& -n_{j, 1} \sum_{j_{2}}\left[\langle\sigma v\rangle_{j, 1 \rightarrow j_{2}}^{\mathrm{CX}} n_{j_{2}, 0}\right] \\
& +n_{j, 0} \sum_{j_{2}} \sum_{q_{2}}\left[\langle\sigma v\rangle_{j_{2}, q_{2} \rightarrow j}^{\mathrm{CX}} n_{j_{2}, q_{2}}\right]-\frac{n_{j, 1}}{\tau_{j, 1}^{P}}, \\
\frac{d n_{j, q}}{d t} & =\left(n_{j, q-1}\langle\sigma v\rangle_{j, q-1}^{I}-n_{j, q}\langle\sigma v\rangle_{j, q}^{I}\right) n_{e} \\
& +n_{j, q+1} \sum_{j_{2}}\left[\langle\sigma v\rangle_{j, q}^{\mathrm{CX}}{ }_{1+1 \rightarrow j_{2}} n_{j_{2}, 0}\right] \\
& \quad-n_{j, q} \sum_{j_{2}}\left[\langle\sigma v\rangle_{j, q \rightarrow j_{2}}^{\mathrm{CX}} n_{j_{2}, 0}\right]-\frac{n_{j, q}}{\tau_{j, q}},
\end{aligned}
$$

$$
\begin{aligned}
\frac{d n_{j, A_{j}}}{d t}= & n_{j, A_{j}-1}\langle\sigma v\rangle_{j, A_{j}-1}^{I} n_{e} \\
& -n_{j, A_{j}} \sum_{j_{2}}\left[\langle\sigma v\rangle_{j, A_{j} \rightarrow j_{2}}^{\mathrm{CX}} n_{j_{2}, 0}\right]-\frac{n_{j, A_{j}}}{\tau_{j, A_{j}}^{P}} .
\end{aligned}
$$

The above set of ion particle balance equations calculates the CSD in the plasma core. For comparison with a CSD as measured experimentally by a Faraday cup, the ion extraction current CSD must be calculated as [1]

$$
\begin{gathered}
j_{j, q}^{\mathrm{ex}}=\frac{e q n_{j, q}}{\tau_{j, q}^{P}} \frac{V_{p}}{\pi r_{p}^{2}}=\frac{e q n_{j, q}}{\tau_{j, q}^{P}} L_{p}, \\
I_{i}^{\mathrm{FC}}=\eta^{\mathrm{FC}} A_{\mathrm{ex}} j_{i}^{\mathrm{ex}}=\eta^{\mathrm{FC}} A_{\mathrm{ex}} \sum_{j} \sum_{q=1}^{A_{j}} j_{j, q}^{\mathrm{ex}},
\end{gathered}
$$

where $r_{p}$ is the plasma radius, $A_{\mathrm{ex}}$ is the area of the extraction aperture, and $\eta^{\mathrm{FC}}$ is the detection efficiency of the Faraday cup.

\section{Fokker-Planck electron model}

The non-Maxwellian anisotropic electron distribution function $f_{e}(v, \theta)$ can be determined by solving the Fokker-Planck equation

$$
\begin{aligned}
\frac{\partial f_{e}}{\partial t} & +\vec{v} \cdot \frac{\partial f_{e}}{\partial \vec{x}}+\frac{\vec{F}}{m_{e}} \cdot \frac{\partial f_{e}}{\partial \vec{v}} \\
& =\left(\frac{\partial f_{e}}{\partial t}\right)_{\mathrm{coll}}+C_{\mathrm{FP}}^{S}(v, \theta)+C_{\mathrm{FP}}^{\mathrm{rf}}(v, \theta),
\end{aligned}
$$

where $\left(\partial f_{e} / \partial t\right)_{\text {coll }}$ is the Fokker-Planck collisional operator, $C_{\mathrm{FP}}^{S}$ is the cold electron source term, and $C_{\mathrm{FP}}^{\mathrm{rf}}$ is the quasilinear ECRF heating term

$$
\begin{gathered}
C_{\mathrm{FP}}^{\mathrm{rf}}=\frac{1}{v_{\perp}} \frac{\partial}{\partial v_{\perp}}\left(v_{\perp} D_{\mathrm{rf}} \frac{\partial f_{e}}{\partial v_{\perp}}\right) . \\
D_{e}^{\mathrm{rf}}=D_{e 0}^{\mathrm{rf}} \exp \left(-v^{2} / 2 c^{2}\right), \\
P_{e}^{\mathrm{rf}}=2 n_{e} m_{e} D_{e 0}^{\mathrm{rf}} V_{p},
\end{gathered}
$$

where $D_{e 0}^{\mathrm{rf}}$ is the $\mathrm{rf}$ diffusion coefficient, $D_{e}^{\mathrm{rf}}$ is the $\mathrm{rf}$ diffusion coefficient adjusted for the smaller effect rf has on electrons with higher energies, and $P_{e}^{\mathrm{rf}}$ is the total $\mathrm{rf}$ power absorbed by the plasma electrons in the plasma volume $V_{p}$.

The nonlinear multispecies Fokker-Planck code FPPAC94 [11] has been incorporated into the model. The Fokker-Planck code also determines the $e-i$ collisional energy exchange rate $p_{j}^{e i}$ and the electron end loss/ confinement time.

In the Fokker-Planck modeling each ion atomic species is treated as a Maxwellian background of density

$$
n_{j}=\sum_{q=1}^{A_{j}} n_{j, q},
$$


and charge

$$
z=\left(\frac{\sum_{q=1}^{A_{j}} n_{j, q} q^{2}}{n_{i}}\right)^{1 / 2} .
$$

This average ion charge for collisions is derived from the general form for the collision frequency of species $a$ with a Maxwellian background species $b$,

$$
\nu_{a}^{b}=\frac{1}{4 \pi \varepsilon_{o}^{2}} \frac{n_{b} e^{4} q_{a}^{2} q_{b}^{2} \ln \Lambda_{a}^{b}}{m_{a}\left(\overline{\boldsymbol{v}}_{a}^{b}\right)^{3}}\left(\frac{m_{a}+m_{b}}{m_{a} m_{b}}\right),
$$

and ignores the difference in $\ln \Lambda$, the Coulomb lambda, for different charge states of the same atomic species. Collisions with neutral particles are ignored in the FokkerPlanck modeling.

The electron end loss is determined by cutting out a loss cone from the electron distribution after each iteration of the Fokker-Planck code. This method was chosen over using the loss cone as a boundary condition to enable calculation of asymmetric end loss flux due to asymmetry mirror fields. Both magnetic mirror and potential confinement are included when determining the loss cone.

As the electron distribution function is highly nonMaxwellian and anisotropic, the electron impact ionization reaction-rate coefficients should be calculated explicitly from distribution functions of the two colliding species,

$$
\begin{aligned}
\langle\sigma v\rangle= & \frac{1}{n_{a} n_{b}} \int d v \int d v^{\prime} f_{a}(v) f_{b}\left(v^{\prime}\right) \\
& \times \sigma\left(\left|v-v^{\prime}\right|\right)\left|v-v^{\prime}\right|
\end{aligned}
$$

The model incorporates a routine [12] to compute the reaction rates for arbitrarily shaped distribution functions. The routine was modified to employ a nonuniformly spaced velocity distribution, suitable to an ECRIS, where the electrons, ions, and neutrals can have average velocities orders of magnitude apart.

\section{RESULTS}

\section{A. Lotz versus Müller cross sections}

As mentioned briefly earlier, we have a choice of empirical formulas to use for the ionization cross section. Figure 1 shows a comparison between Ne plasma CSD predictions when using the Lotz or Müller formulas. The Müller formula produces a sharp peak in the CSD two charge states below the fully stripped level, that is not evident when using the Lotz formula. Presumably, this peak is a result of Müller's formula ignoring the effects of electron subshells below the outermost subshell. This peak is not evident in typical ANL-ECRII experimental data such as shown in Fig. 2. Thus, the Lotz formula appears to be more appropriate for ECRIS modeling and will be used for the remainder of this paper.

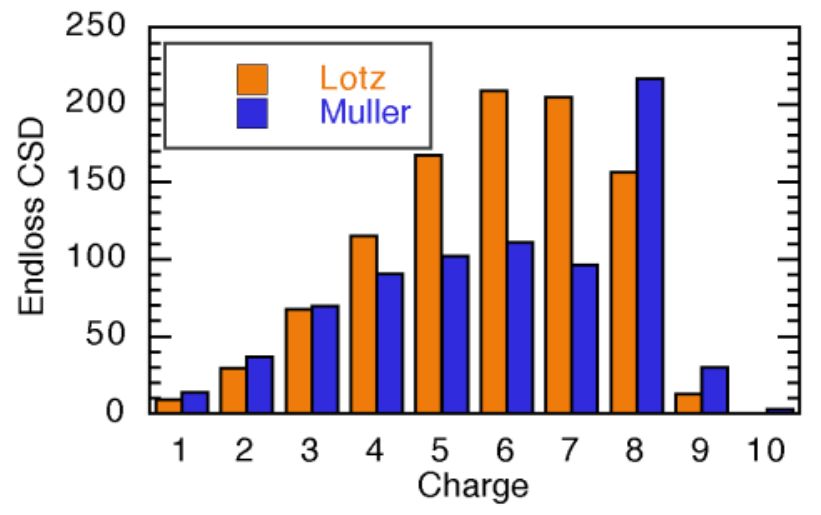

FIG. 1. (Color) Comparison of CSD predictions for a pure $\mathrm{Ne}$ plasma when using the Lotz or Müller formulas for an electron collisional ionization cross section.

\section{B. CSD with electron Fokker-Planck modeling}

To investigate the validity of the model, comparisons have been made with Faraday cup measurements from ECRII [13] at Argonne. The experimental data are shown as distribution $a$ in Fig. 2.

The need for Fokker-Planck electron modeling is demonstrated by distributions $b$ and $c$ in Fig. 2. CSD $b$ was produced by a Maxwellian electron distribution (with a loss cone) of $T_{e}=1.5 \mathrm{keV}$ while CSD $c$ results from combining 50\% nearly collisionless hot electrons at $100 \mathrm{keV}$ with $50 \%$ warm electrons at $1.5 \mathrm{keV}$. Clearly, one can match the experimental data using very different assumed electron distributions.

To eliminate this arbitrariness in favor of a unique solution, the electron distribution should be determined by solving the Fokker-Planck equation. The predictions of the Fokker-Planck electron modeling are given as CSD $d$ in Fig. 2. The Fokker-Planck electron modeling produces a good match to the experimental data with less arbitrariness. The electron "temperature" predicted by the Fokker-Planck modeling is $T_{e}=\frac{2}{3}\left\langle E_{e}\right\rangle=36 \mathrm{keV}$. The actual electron distribution functions used in calculating the CSDs shown in Fig. 2 are given in Fig. 3.

\section{DISCUSSION}

To be considered completely predictive, the model should rely on measured experimental knobs. Even with Fokker-Planck modeling of the electrons, some quantities such as the core plasma length and the electron confining potential still need to be arbitrarily input to the model. By extending the modeling spatially to 1D axially, one should be able to determine some of these quantities from the plasma confinement. Accounting for spatial variations in plasma parameters, magnetic field and the electrostatic potential should also improve the confinement and CSD modeling of an ECRIS. 


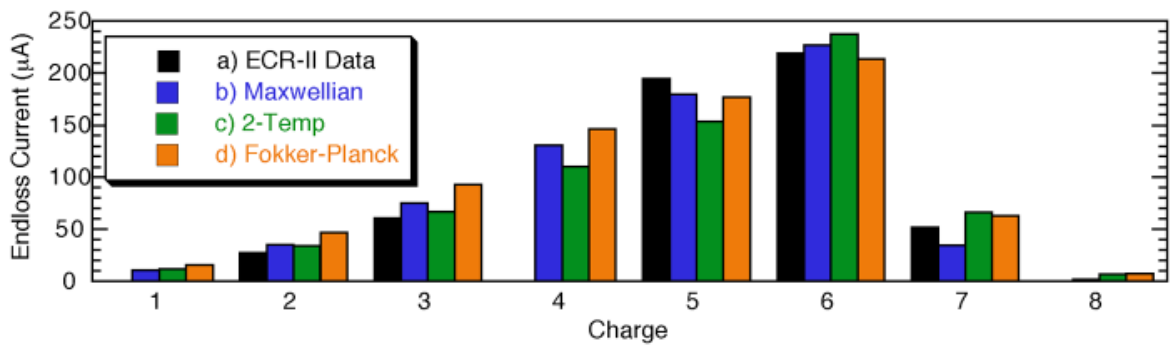

FIG. 2. (Color) Comparison of modeling results with ANL-ECRII Faraday cup measurements.

Because of the high electron mobility, the electron spatial effects can be accounted for by a bounce-averaged Fokker-Planck code. A bounce-averaged Fokker-Planck treatment should also improve the ECRH modeling by taking into account the location of the ECR resonance. This location restricts the perpendicular heating of electrons to only those whose pitch angle allows them to reach the resonant surface.

In a typical ECRIS, however, the ion bounce frequency is much smaller than the ion collision frequency and a bounce-averaged treatment is inappropriate. Extending a fluid model, axially from the plasma core, may also be inappropriate as the plasma near the extraction point will be less dense and the ions will be accelerating down the plasma sheath. The edge ions will be much
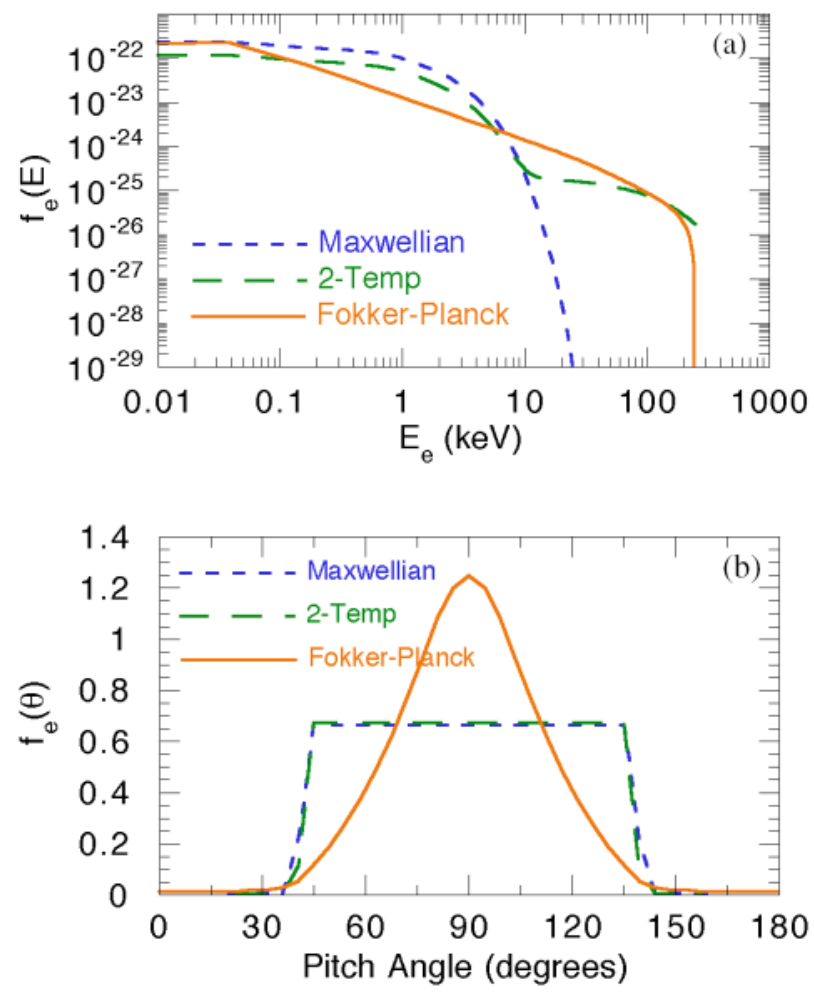

FIG 3. (Color) Electron distribution functions corresponding to the CSDs of Fig. 2. (a) is the distribution integrated over pitch angle $f_{e}(v)$ and (b) is the distribution integrated over velocity $f_{e}(\theta)$. less collisional than in the center of the plasma and the fluid approximations may not apply. The Monte Carlo method [14] is better suited for determining the ion spatial effects. This method can handle both highly collisional and collisionless regimes with smoothly varying and nonsymmetric magnetic fields or potentials.

The simple step neutral modeling of the 0D model will need to be replaced to extend the model to $1 \mathrm{D}$. The simple step model can easily be extended to 1D using coupled balance equations similar to Eq. (1) for each axial zone, but this method greatly oversimplifies the neutral modeling. A 3D Monte Carlo neutral model would be ideal as the neutrals are not constrained to follow magnetic geometry. However, the improved accuracy of such a model is doubtful given the uncertainties of the 3D plasma parameter profiles in a multipole mirror geometry.

\section{ACKNOWLEDGMENTS}

The authors thank Dr. M. Rensink, Dr. B. Stallard, Dr. T. Rognlien, and Dr. L. LoDestro for their help with this work. The work at FARTECH was supported by the U.S. DOE-SBIR Grant No. DE-FG03-97ER82381. The work at Argonne National Laboratory was supported by the U.S. Department of Energy, Nuclear Physics Division, under Contract No. W-31-109-ENG-38.

[1] H. I. West, National Technical Information Service, Report No. UCRL-53391, 1982.

[2] G. Shirkov, Nucl. Instrum. Methods Phys. Res., Sect. A 322, 161 (1992).

[3] V.D. Dougar-Jabon, A.M. Umnov, and V.B. Kutner, Rev. Sci. Instrum. 67, 1152 (1996).

[4] K. S. Golovanisky and G. Melin, Rev. Sci. Instrum. 63, 2886 (1992).

[5] W. Lotz, Z. Phys. 206, 205 (1967).

[6] A. Müller, E. Salzborn, R. Frodl, R. Becker, H. Klein, and H. Winter, J. Phys. B 13, 1877 (1980).

[7] A. Müller and R. Frodl, Phys. Rev. Lett. 44, 29 (1980).

[8] A. Müller and E. Salzborn, Phys. Lett. 62A, 391 (1977).

[9] G. Shirkov, CERN Report No. CERN/PS 94-13 (HI), 1994.

[10] T.D. Rognlien and T.A. Cutler, Nucl. Fusion 20, 1003 (1980). 
[11] A.A. Mirin, M. G. McCoy, G.P. Tomaschke, and J. Killeen, Comput. Phys. Commun. 81, 403 (1994).

[12] A. A. Mirin and M. G. McCoy, Comput. Phys. Commun. 51, 369 (1988).

[13] M. Schlapp, R.C. Pardo, R.C. Vondrasek, J. Szczech,
P. J. Bilquist, J. Vieregg, Z. Q. Xie, C. M. Lyneis, and R. Harkewicz, Rev. Sci. Instrum. 69, 631 (1998).

[14] J. Spanier and E. M. Gelbard, in Monte Carlo Principles and Neutron Transport Problems (Addison-Wesley, Reading, PA, 1969). 